\title{
The generalized SH-wave equation
}

\author{
José M. Carcione* and Fabio Cavallini*
}

\begin{abstract}
We present a generalization of the $S H$-wave equation for anisotropic and dissipative media. The most general case in which $S H$-waves are decoupled from $P$ - and $S V$-waves at all propagation angles is that of propagation in the plane of symmetry of a monoclinic medium. In the isotropic case, the $S H$ constitutive equation involves only one elastic coefficient (the rigidity); here, three elastic coefficients are needed. Moreover, dissipation is introduced by using Boltzmann's law based on several relaxation mechanisms. Anisotropic attenuation and velocity dispersion are guaranteed by choosing different relaxation functions for the principal axes. The wave equation, in the displacement and velocity-stress formulations, is solved by using time-domain spectral modeling techniques. The snapshots and seismograms of the computed displacement field show attenuation and anisotropy effects on traveltime (mainly because of anisotropy) and amplitude (because of combined anisotropy and dissipation), in agreement with the theoretical patterns of energy velocity and attenuation curves.
\end{abstract}

\section{INTRODUCTION}

The observed attenuation and anisotropy of seismic waves is an important source of information regarding the composition and structural features of hydrocarbon reservoirs. In particular, the presence of partially saturated aligned cracks induces directional dissipation because of energy losses by the anisotropic flow of the viscous fluid (Hudson, 1990). The same effect is verified in finely layered systems (Chevalier, 1988; Carcione, 1992a). Indeed, for seismic wavelengths the medium behaves as an effective anisotropic material where attenuation generally depends on the wave-propagation direction. Previous works attempting to simulate anisotropic waves including attenuation effects are attributed to Carcione (1990) who solved the $q P-q S V$ full-wave equation by spectral techniques, Gajewski and Pšenčík (1992) who used the ray method for weakly anisotropic media and, more recently, Le et al. (1994) who simulated $S H$-waves by complex ray tracing.

Propagation in the plane of mirror symmetry of a monoclinic medium includes a shear wave with polarization perpendicular to the plane (antiplane strain motion). This is the most general situation for which pure shear waves exist at all propagation angles (e.g., pure shear-wave propagation in hexagonal media is a degenerate case). A set of parallel fractures embedded in a transversely isotropic formation can be represented by a monoclinic medium. When the plane of mirror symmetry of this medium is vertical, the pure antiplane strain waves are $S H$-waves. Moreover, monoclinic media include many other cases of higher symmetry. Weakly tetragonal media, strongly trigonal media, and orthorhombic media are subsets of the set of monoclinic media.

A realistic rheological equation of the monoclinic medium should include anelastic effects, since a sedimentary formation itself is anelastic, and fractures induce intrinsic attenuation when they are filled with fluids. The result is that velocity dispersion alters the shape of the wavefront (an ellipse in the elastic case), and that the amplitude of the signal is not isotropic along the wavefront as a result of anisotropic dissipation. The constitutive equation proposed here includes two relaxation functions describing the anelastic properties along the vertical and horizontal directions, respectively. These are the generalizations of the elastic stiffnesses $c_{44}$ and $c_{66}$, while $c_{46}$ plays the role of a geometric parameter as in the elastic case. The convolutional integrals are circumvented by the introduction of two sets of memory variables, each representing different dissipation mechanisms. The resulting wave equation for the displacement field is solved by using spectral techniques in the time domain. The problem is basically 2-D if a line source of shear waves with polarization normal to the symmetry plane is assumed.

\section{THE CONSTITUTIVE EQUATION}

Let us assume that the $(x, z)$ plane is the symmetry plane of a monoclinic medium. The anti-plane assumption that

Manuscript received by the Editor July 2, 1993; revised manuscript received July 7, 1994.

* Osservatorio Geofisico Sperimentale, P.O. Box 2011 Opicina, 34016 Trieste, Italy.

(C) 1995 Society of Exploration Geophysicists. All rights reserved. 
displacement $\mathbf{u}=u(x, z, t) \hat{e}_{y}$ implies that the only nonzero stress components are $\sigma_{y z}(x, z, t)$ and $\sigma_{x y}(x, z, t)$. In condensed notation, the stress-strain relation for pure shear waves in this plane is:

$$
\left[\begin{array}{l}
\sigma_{y z} \\
\sigma_{x y}
\end{array}\right]=\left[\begin{array}{ll}
\psi_{44} & \psi_{46} \\
\psi_{46} & \psi_{66}
\end{array}\right] *\left[\begin{array}{l}
\dot{\gamma}_{y z} \\
\dot{\gamma}_{x y}
\end{array}\right]
$$

where $\gamma_{y z}(x, z, t)$ and $\gamma_{x y}(x, z, t)$ are the strain components, and the $\psi_{I J}(x, z, t)$ are relaxation components. The symbol ${ }^{*}$ represents time convolution and a dot above a variable denotes time differentiation. The strains can be expressed in terms of the displacement $u$, normal to the plane of symmetry, as:

$$
\gamma_{y z}=\frac{\partial u}{\partial z}, \text { and } \quad \gamma_{x y}=\frac{\partial u}{\partial x}
$$

Equation (1) is quite general within the framework of linear viscoelasticity. The problem now is the choice of the time dependence of the relaxation components, which should be as general as possible to describe anisotropic attenuation and a $Q$ factor with an arbitrary frequency dependence. We know that $\psi_{44}$ describes the properties of the shear wave along the $z$-direction (say, the vertical direction), and that $\psi_{66}$ is related to the horizontal direction. In fact, in the elastic case the wavefront curve is an ellipse whose values along the vertical and horizontal directions are $\sqrt{c_{66} / \rho}$ and $\sqrt{c_{44} / \rho}$, respectively, where the $c_{I J}(x, z)$ are the elastic stiffnesses and $\rho(x, z)$ is the density (Schoenberg and Costa, 1991). Following Carcione (1990) we take

$$
\begin{array}{r}
\psi_{44}(t)=c_{44} \psi^{(1)}(t) H(t), \quad \psi_{66}(t)=c_{66} \psi^{(2)}(t) H(t) \\
\text { and } \psi_{46}(t)=c_{46} H(t),
\end{array}
$$

where

$$
\begin{aligned}
\psi^{(m)}(t)=\left[1-\frac{1}{L^{(m)}} \sum_{\ell=1}^{L^{(m)}}\left(1-\frac{\tau_{\varepsilon \ell}^{(m)}}{\tau_{\boldsymbol{\sigma} \ell}^{(m)}}\right) e^{-t / \tau} \underset{\sigma \ell}{(m)}\right], & \\
& m=1,2,
\end{aligned}
$$

and $H(t)$ is the Heaviside function. The quantities $\tau_{\varepsilon \ell}^{(m)}$ and $\tau_{\sigma \ell}^{(m)}$ are material relaxation times. Equation (4) is the relaxation function of $L^{(m)}$ standard linear solid elements connected in parallel (Casula and Carcione, 1992). Each element or pair of relaxation times describes a particular dissipation mechanism. Anisotropy of the anelastic properties (attenuation and velocity dispersion) is guaranteed by the choice of two different relaxation functions, $m=1$ for the vertical direction and $m=2$ for the horizontal direction [instead, Carcione (1990) considers only one relaxation function to model the anelastic properties of the $S H$-wave].

\section{THE WAVE EQUATION}

After substitution of the relaxation components (3) and using properties of the convolution, the constitutive equation (1) can be rewritten as:

$\left[\begin{array}{l}\sigma_{y z} \\ \sigma_{x y}\end{array}\right]=\left[\begin{array}{ll}\hat{c}_{44} & c_{46} \\ c_{46} & \hat{c}_{66}\end{array}\right]\left[\begin{array}{l}\gamma_{y z} \\ \gamma_{x y}\end{array}\right]+\left[\begin{array}{cc}c_{44} \dot{\psi}^{(1)} H & 0 \\ 0 & c_{66} \dot{\psi}^{(2)} H\end{array}\right] *\left[\begin{array}{l}\gamma_{y z} \\ \gamma_{x y}\end{array}\right]$, where the first term is the unrelaxed or high-frequency response of the medium and the second term contains the history of deformation of the material. The unrelaxed elastic stiffnesses are given by $\hat{c}_{44}=c_{44} \psi^{(1)}(0)$ and $\hat{c}_{66}=$ $c_{66} \psi^{(2)}(0)$. To avoid the convolution integrals in equation (5) we define the following memory variables

$$
e_{\ell}^{(1)}=\phi_{\ell}^{(1) *} \gamma_{y z}, \quad \ell=1, \ldots, L^{(1)},
$$

and

$$
e_{\ell}^{(2)}=\phi_{\ell}^{(2) *} \gamma_{x y}, \quad \ell=1, \ldots, L^{(2)}
$$

where

$$
\phi_{\ell}^{(m)}(t)=\frac{H(t)}{L^{(m)} \tau_{\varepsilon \ell}^{(m)}}\left(1-\frac{\tau_{\varepsilon \ell}^{(m)}}{\tau_{\boldsymbol{\sigma} \ell}^{(m)}}\right) e^{-t / \tau} \tau_{\sigma \ell}^{(m)}, \quad m=1,2
$$

is called the response function of the $\ell$ th mechanism. With these definitions, equation (5) takes the form:

$$
\left[\begin{array}{l}
\sigma_{y z} \\
\sigma_{x y}
\end{array}\right]=\left[\begin{array}{ll}
\hat{c}_{44} & c_{46} \\
c_{46} & \hat{c}_{66}
\end{array}\right]\left[\begin{array}{l}
\gamma_{y z} \\
\gamma_{x y}
\end{array}\right]+\left[\begin{array}{cc}
c_{44} & 0 \\
0 & c_{66}
\end{array}\right]\left[\begin{array}{ll}
\sum & e_{\ell}^{(1)} \\
\sum & e_{\ell}^{(2)}
\end{array}\right] .
$$

The response function [equation (7)] satisfies the following differential equation:

$$
\dot{\phi}_{\ell}^{(m)}=-\phi_{\ell}^{(m)} / \tau_{\sigma \ell}^{(m)} .
$$

Using equation (9), time differentiation of the memory variables (6) yields the following first-order equations:

$$
\dot{e}_{\ell}^{(1)}=\phi_{\ell}^{(1)}(0) \gamma_{y z}-e_{\ell}^{(1)} / \tau_{\sigma \ell}^{(1)}, \quad \ell=1, \ldots, L^{(1)},
$$

and

$$
\dot{e}_{\ell}^{(2)}=\phi_{\ell}^{(2)}(0) \gamma_{x y}-e_{\ell}^{(2)} / \tau_{\sigma \ell}^{(2)}, \quad \ell=1, \ldots, L^{(2)} .
$$

To complete the scheme, the description of wave propagation requires the equation of momentum conservation (Ewing et al., 1957),

$$
\frac{\partial \sigma_{y z}}{\partial z}+\frac{\partial \sigma_{x y}}{\partial x}=\rho \ddot{u} .
$$

For instance, when $L^{(1)}=L^{(2)}=1$, the wave equation for the displacement $u$ and variables of strain memory $e^{(1)}$ and $e^{(2)}$ becomes

$$
\begin{aligned}
& \left(c_{44} e^{(1)}+\tau^{(1)} c_{44} u,_{z}+c_{46} u, x\right), z \\
& +\left(c_{66} e^{(2)}+\tau^{(2)} c_{66} u,_{x}+c_{46} u,{ }_{, z}\right), \rho \ddot{u}, \\
& \tau_{\sigma}^{(1)} \dot{e}^{(1)}=\left(1-\tau^{(1)}\right) u_{, z}-e^{(1)}, \\
& \text { and } \tau_{\sigma}^{(2)} \dot{e}^{(2)}=\left(1-\tau^{(2)}\right) u_{, x}-e^{(2)}, \quad \text { (12) }
\end{aligned}
$$

where $\tau^{(m)}=\tau_{\varepsilon}^{(m)} / \tau_{\sigma}^{(m)}$ and abbreviated notation for spatial differentiation is used.

Equation (12) is solved in the time domain by using spectral techniques (Tal-Ezer et al., 1990). The above displacement formulation of the wave equation is solved by using the Fourier pseudospectral method for computing the spatial derivatives. When the problem includes boundary conditions like, for instance, the surface of the earth, the Chebyshev differential operator is used (e.g., Kosloff et al., 
1990; Carcione, 1992b). In this case, the modeling requires a velocity-stress formulation. Defining the particle velocity $v=\dot{u}$, the velocity-stress differential equations read

$$
\begin{gathered}
\rho \dot{v}=\frac{\partial \sigma_{y z}}{\partial z}+\frac{\partial \sigma_{x y}}{\partial x}, \\
\dot{\sigma}_{y z}=\hat{c}_{44} v,_{z}+c_{46} v,_{x}+c_{44} \sum_{\ell=1}^{L^{(1)} \varepsilon^{(1)},} \\
\dot{\sigma}_{x y}=c_{46} v,_{z}+\hat{c}_{66} v, x+c_{66} \sum_{\ell=1}^{L^{(2)}} \varepsilon^{(2)}, \\
\tau_{\sigma}^{(1)} \dot{\varepsilon}^{(1)}=\left(1-\tau^{(1)}\right) v_{,_{z}}-\varepsilon^{(1)},
\end{gathered}
$$

and

$$
\tau_{\boldsymbol{\sigma}}^{(2)} \dot{\boldsymbol{\varepsilon}}^{(2)}=\left(1-\tau^{(2)}\right) v,_{x}-\varepsilon^{(2)},
$$

where $\varepsilon^{(m)} \equiv \dot{e}^{(m)}, m=1,2$. In the isotropic and elastic limit, equations (13a)-(13d) give the conventional velocitystress differential equations (Virieux, 1984).

\section{PLANE-WAVE ANALYSIS}

A brief plane-wave analysis helps one to understand the physics of wave propagation in this type of medium. The analogy with the elastic case is found in Schoenberg and Costa (1991) and the analogy with pure shear-wave propagation in orthorhombic media in Carcione (1994). Let the displacement be associated with the following homogeneous plane wave of slowness $s$ and attenuation $\alpha$ :

$$
u=U_{0} e^{i \omega t} e^{-i(\omega s-i \alpha) \hat{\mathbf{s}} \cdot \mathbf{x}},
$$

where $\omega$ is the angular frequency, $x \equiv(x, z)$ and $(\omega s-i \alpha) \hat{\mathbf{s}}$ $\equiv \mathbf{k}$ is the complex wavenumber vector with $\hat{\mathbf{s}} \equiv\left(\ell_{x}, \ell_{z}\right)$ defining the propagation direction. Substituting this form into equation (1) and the result into the equation of momentum conservation (11) gives the complex dispersion relation

$$
p_{66} \ell_{x}^{2}+2 p_{46} \ell_{x} \ell_{z}+p_{44} \ell_{z}^{2}=\rho(\omega / k)^{2},
$$

where $p_{I J}$ are the complex stiffness components given by the Fourier transform of the time derivatives of the relaxation components. This gives

$p_{44}=c_{44} M^{(1)}, \quad p_{66}=c_{66} M^{(2)}$ and $p_{46}=c_{46}$,

where

$$
M^{(m)}(\omega)=\frac{1}{L^{(m)}} \sum_{\ell=1}^{L^{(m)}} \frac{1+i \omega \tau_{\varepsilon \ell}^{(m)}}{1+i \omega \tau_{\sigma \ell}^{(m)}}, \quad m=1,2,
$$

are the dimensionless complex moduli. Since the slowness $s$ is equal to $\operatorname{Re}[k / \omega]$, we obtained from equation (15) that the slowness curve is

$$
\operatorname{Re}\left[\left(\frac{s_{x}^{2}}{\rho / p_{66}}+\frac{2 s_{x} s_{z}}{\rho / p_{46}}+\frac{s_{z}^{2}}{\rho / p_{44}}\right)^{-1 / 2}\right]-1=0,
$$

where $s_{x}=s \ell_{x}$ and $s_{z}=s \ell_{z}$. Note that $\hat{\mathbf{s}}=(0,1)$ implies $s=\operatorname{Re}\left[\sqrt{\rho / p_{44}}\right]$, and $\hat{\mathbf{s}}=(1,0)$ implies $s=\operatorname{Re}\left[\sqrt{\rho / p_{66}}\right]$; hence, the velocity dispersion is determined by $M^{(1)}$ along the vertical direction and by $M^{(2)}$ along the horizontal direction. Limiting cases of equation (18) are equation (6) of Schoenberg and Costa (1991) (monoclinic elastic) and equation (45) of Carcione (1994) (orthorhombic viscoelastic).

The velocity of the energy (wavefront) is defined by the average power flow density divided by the average energy density. Following the same calculations as given by Carcione (1994), we get

$$
\begin{aligned}
\mathbf{V}_{e}= & \frac{V_{p}}{\operatorname{Re}[V]}\left\{\operatorname { R e } \left[\frac { 1 } { \rho V } \left[\left(p_{66} \ell_{x}+p_{46} \ell_{z}\right) \hat{\mathbf{e}}_{x}\right.\right.\right. \\
& \left.\left.\left.+\left(p_{44} \ell_{z}+p_{46} \ell_{x}\right) \hat{\mathbf{e}}_{z}\right]\right]\right\},
\end{aligned}
$$

where $V=\omega / k$, and $V_{p}=1 / \operatorname{Re}(1 / V)$ is the phase velocity. Compare equation (19) to equation (A-13) of Schoenberg and Costa (1991) and to equation (37) of Carcione (1994). It can be shown that the energy velocity, and therefore the wavefront, depends only on $M^{(2)}$ and $M^{(1)}$ in the vertical and horizontal directions, respectively.

The quality factor is defined as the ratio of strain energy density to dissipated energy density. It has the following simple form (Carcione and Cavallini, 1992)

$$
Q=-\frac{\operatorname{Re}\left[k^{2}\right]}{\operatorname{Im}\left[k^{2}\right]} .
$$

Since the attenuation is given by $\alpha=-\operatorname{Im}[k]$, it is easy to show that $\alpha=\left(\sqrt{Q^{2}+1}-Q\right) \omega s$. The values of $Q$ along the vertical and horizontal directions are $\operatorname{Re}\left[M^{(2)}\right] / \operatorname{Im}\left[M^{(2)}\right]$ and $\operatorname{Re}\left[M^{(1)}\right] / \operatorname{Im}\left[M^{(1)}\right]$, respectively. These relations allow a close control of the shape of the attenuation curves.

\section{EXAMPLE}

We consider a monoclinic medium with $p_{44}=c_{44} M^{(1)}$, $p_{66}=c_{66} M^{(2)}$, and $p_{46}=c_{46}$, where

$$
M^{(m)}(\omega)=\frac{1+i \omega \tau_{\varepsilon}^{(m)}}{1+i \omega \tau_{\sigma}^{(m)}}, \quad m=1,2 .
$$

In the time domain, the wave equation (12) corresponds to this rheology. The quality factors for homogeneous waves along the $z$-and $x$-axes are

$$
Q^{(m)}(\omega)=Q_{0}^{(m)} \frac{1+\omega^{2} \tau_{0}^{(m) 2}}{2 \omega \tau_{0}^{(m)}}, \quad m=1,2,
$$

respectively, where

$$
Q_{0}^{(m)}=\frac{2 \tau_{0}^{(m)}}{\tau_{\varepsilon}^{(m)}-\tau_{\sigma}^{(m)}},
$$

and

$$
\tau_{0}^{(m)}=\sqrt{\tau_{\varepsilon}^{(m)} \tau_{\sigma}^{(m)}} .
$$

The curve $Q^{(m)}(\omega)$ has its peak at $\omega_{0}^{(m)}=1 / \tau_{0}^{(m)}$, and the value of $Q^{(m)}(\omega)$ at the peak is $Q_{0}^{(m)}$. The low-frequency elastic stiffnesses are taken as: $c_{44}=10.0 \mathrm{GPa}, c_{66}=$ $22.5 \mathrm{GPa}$ and $c_{46}=5.0 \mathrm{GPa}$, and the density as 
$\rho=2500 \mathrm{~kg} / \mathrm{m}^{3}$. The relaxation peaks of both dissipation mechanisms are centered at $f_{0}=1 / 2 \pi \tau_{0}^{(m)}=25 \mathrm{~Hz}$, and the values of the peak quality factors are $Q_{0}^{(1)}=20$ and $Q_{0}^{(2)}=$ 100.

Figure 1 displays polar diagrams of the attenuation and energy velocity at a frequency of $25 \mathrm{~Hz}$. The orientation and shape of the curves depend on the elasticities and the dissipation mechanisms, which are intrinsic properties of the

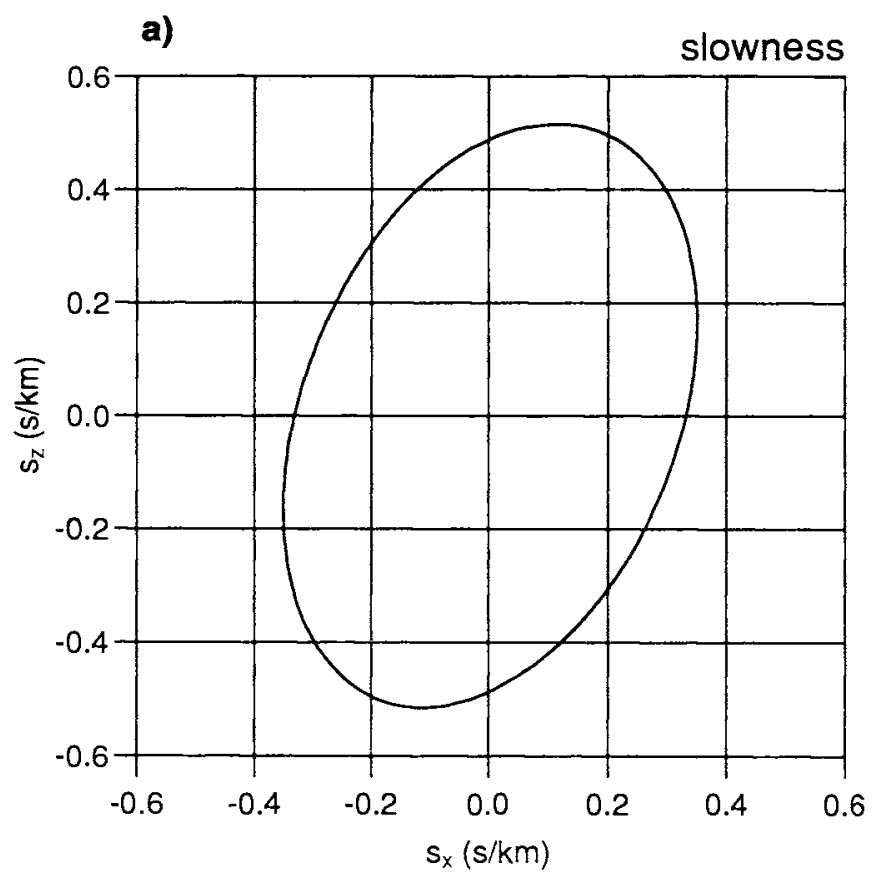

c)

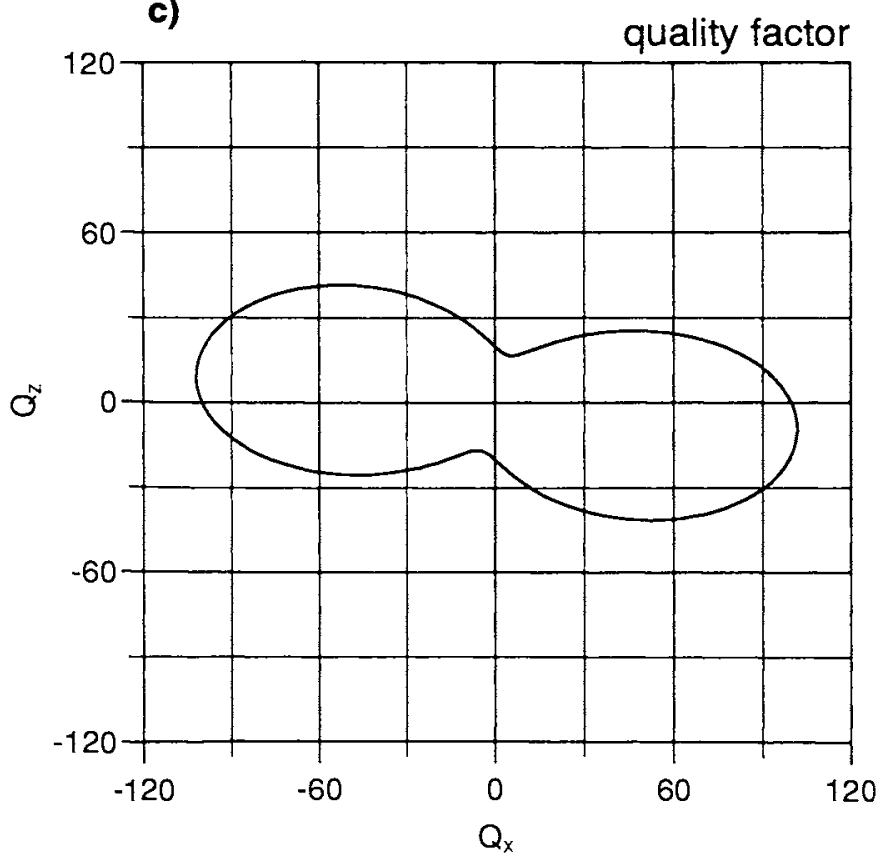

medium, and the direction of propagation. In particular, the dissipation is strong in the vertical direction.

In the following figures, we consider a flat interface where the upper half-space is a medium with monoclinic (or higher) symmetry, and the lower half-space is an elastic isotropic medium with $c_{44}=c_{66}=48 \mathrm{GPa}$ and $\rho=3000 \mathrm{~kg} / \mathrm{m}^{3}$. Figure 2 shows snapshots (not scaled) of the displacement wavefields produced by a line source with central frequency

b)

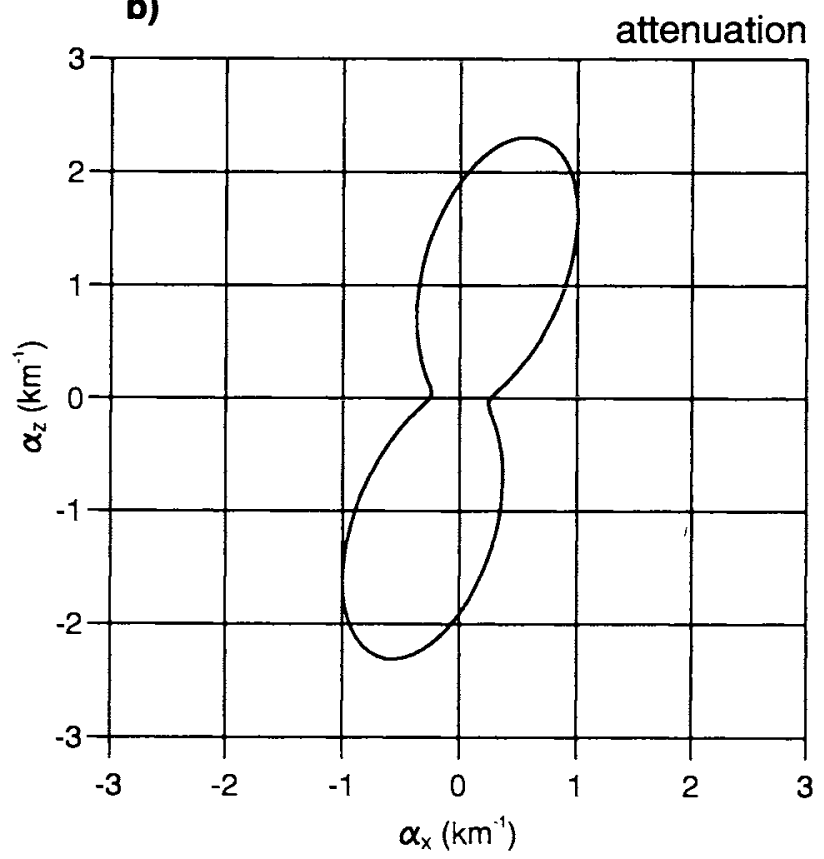

d)

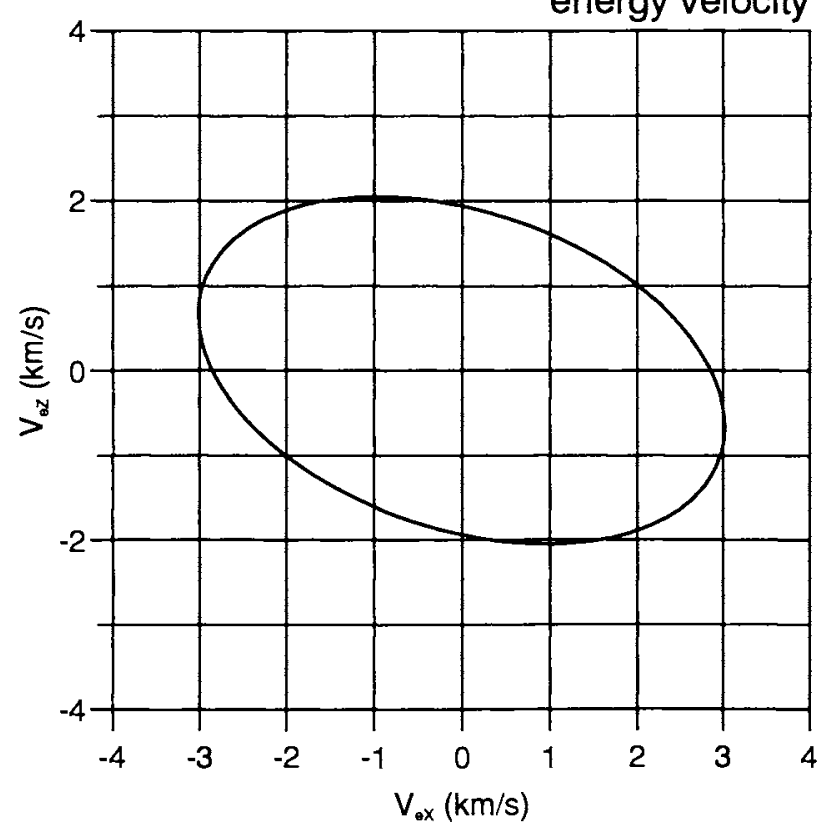

FIG. 1. Polar representations of (a) slowness, (b) attenuation, (c) quality factor, and (d) energy velocity for a homogeneous wave in the symmetry plane of a monoclinic medium. The curves correspond to a frequency of $25 \mathrm{~Hz}$. 
of $25 \mathrm{~Hz}$. The upper medium is isotropic-elastic (left column), anisotropic-elastic (central column), or anisotropicviscoelastic (right column). In the left column, the elastic stiffnesses of the upper medium are $c_{66}=c_{44}=10 \mathrm{GPa}$ and $c_{46}=0$. As can be observed, a comparison of the first elastic and viscoelastic snapshots (taken from the left) shows that the wave is more attenuated in the vertical direction, as predicted by the theoretical curves. Moreover, the reflected field in the anelastic case is clearly weaker than in the elastic case (see snapshots at $t=340 \mathrm{~ms}$ ). An important effect is the change in amplitude along the wavefront, as can be appreciated in the last snapshots. This is caused by the anisotropic-dissipation properties of the monoclinic medium, and by a reflection coefficient presumably different from that of the elastic case.

The corresponding seismograms are illustrated in Figure 3. Anelasticity and anisotropy affect the reflected wave more than the direct wave, as shown by the different amplitude variation with offset of the reflected event in the three cases. Finally, reflection traveltimes are considerably different in the isotropic and anisotropic cases.

\section{CONCLUSIONS}

We have developed a wave equation for simulating viscoelastic pure shear waves in a monoclinic medium. The theory includes other cases of higher symmetry, like orthorhombic and transversely isotropic media. As is well known (e.g., Winterstein, 1990), these rheologies describe a set of systems (cracked formations, fine layering, etc.) frequently appearing in reservoir environments. A frequency-domain analysis shows how the values of the attenuation (or the quality factor) can be chosen along the horizontal and vertical directions for modeling purposes. The use of a simple wave equation that considers the anisotropy of the elastic constants and directional dissipation can be convenient for analyzing the performance of amplitude variations with offset in the presence of anisotropy and attenuation. Indeed, in the case of a reservoir environment, an interpretation of the seismic response based merely on the assumption of elastic and isotropic medium is often adequate to give an indication of the location of the reservoir, but a correct diagnosis of its composition and structural features requires the consideration of the directional dissipation effects. Finally, from the point of view of research, the modeling algorithm may prove helpful for studying the properties of inhomogeneous viscoelastic body waves generated at anelastic-anelastic interfaces.

\section{ACKNOWLEDGMENTS}

This work was funded in part by the European Commission in the framework of the JOULE programme, subprogramme Advanced Fuel Technologies. The authors are grateful to R. J. Brown for his careful review of the paper, with useful technical suggestions.

\section{REFERENCES}

Carcione, J. M., 1990, Wave propagation in anisotropic linear viscoelastic media: Theory and simulated wavefields: Geophys. J. Int., 101, 739-750.

1992a, Anisotropic $Q$ and velocity dispersion of finely layered media: Geophys. Prosp., 40, 761-783.

$1992 \mathrm{~b}$, Modeling anelastic singular surface waves in the earth: Geophysics, 57, 781-792.

-1994, Wavefronts in dissipative anisotropic media: Geophysics, 59, 644-657.

Carcione, J. M., and Cavallini, F., 1992, Attenuation and quality factor surfaces in anisotropic-viscoelastic media: Mech. of Mat., 19 , no. 4, 311-327.

Casula, G., and Carcione, J. M., 1992, Generalized mechanical model analogies of linear viscoelastic behaviour: Boll. Geofis. Teor. Appl., 34, 235-256.

Chevalier, Y., 1988, Dispersion of harmonic waves in elastic and viscoelastic periodic composite materials, in Parker, D. F., and Maugin, G. A., Eds., Recent developments in surface acoustic waves: Springer-Verlag, 260-268.

Ewing, W. M., Jardetzky, W. S., and Press, F., 1957, Elastic waves in layered media: McGraw-Hill Book Co.

Gajewski, D., and Pšenčík, I., 1992, Vector wavefields for weakly attenuating anisotropic media by the ray method: Geophysics, 57, $27-38$.

Hudson, J. A., 1990, Attenuation due to second order scattering in material containing cracks: Geophys. J. Internat., 102, 485-490.

Kosloff, D., Kessler, D., Queiroz Filho, A., Tessmer, E., Behle, A., and Strahilevitz, R., 1990 , Solution of the equations of dynamic elasticity by a Chebychev spectral method: Geophysics, 55, 734-748.

Le, L. H. T., Krebes, E. S., and Quiroga-Goode, G. E., 1994, Synthetic seismograms for $S H$ waves in anelastic transversely isotropic media: Geophys. J. Internat., 116, 598-604.

Schoenberg, M., and Costa, J., 1991, The insensitivity of reflected $S H$ waves to anisotropy in an underlying layered medium: Geophys. Prosp., 39, 985-1003.

Tal-Ezer, H., Carcione, J. M., and Kosloff, D., 1990, An accurate and efficient scheme for wave propagation in linear viscoelastic media: Geophysics, 55, 1366-1379.

Virieux, J., 1984, $S H$-wave propagation in heterogeneous media: Velocity-stress finite-difference method: Geophysics, 49, 19331957.

Winterstein, D. F., 1990, Velocity anisotropy terminology for geophysicists: Geophysics, 55, 1070-1088. 
554

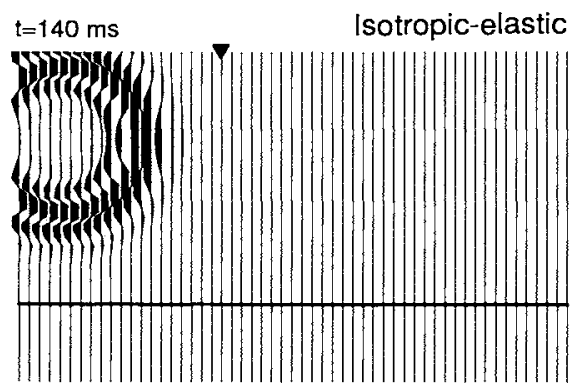

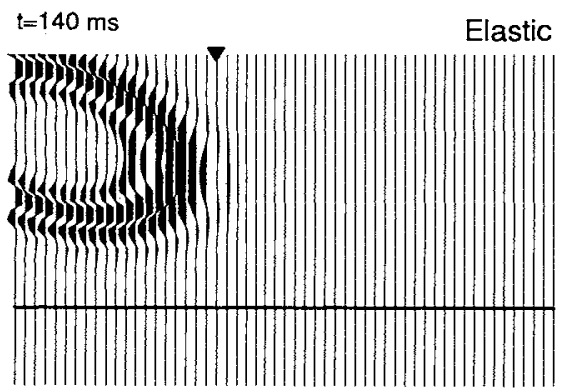
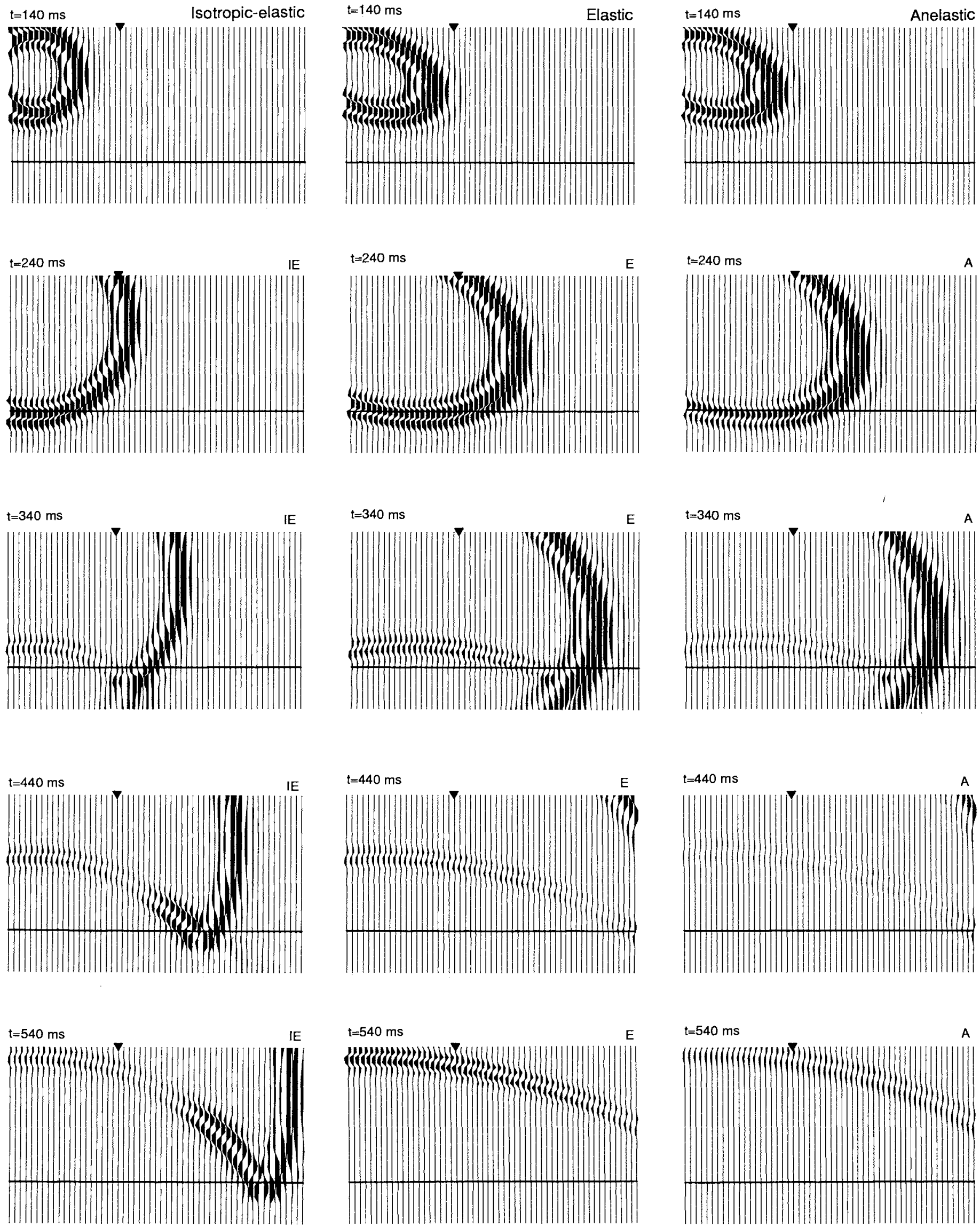

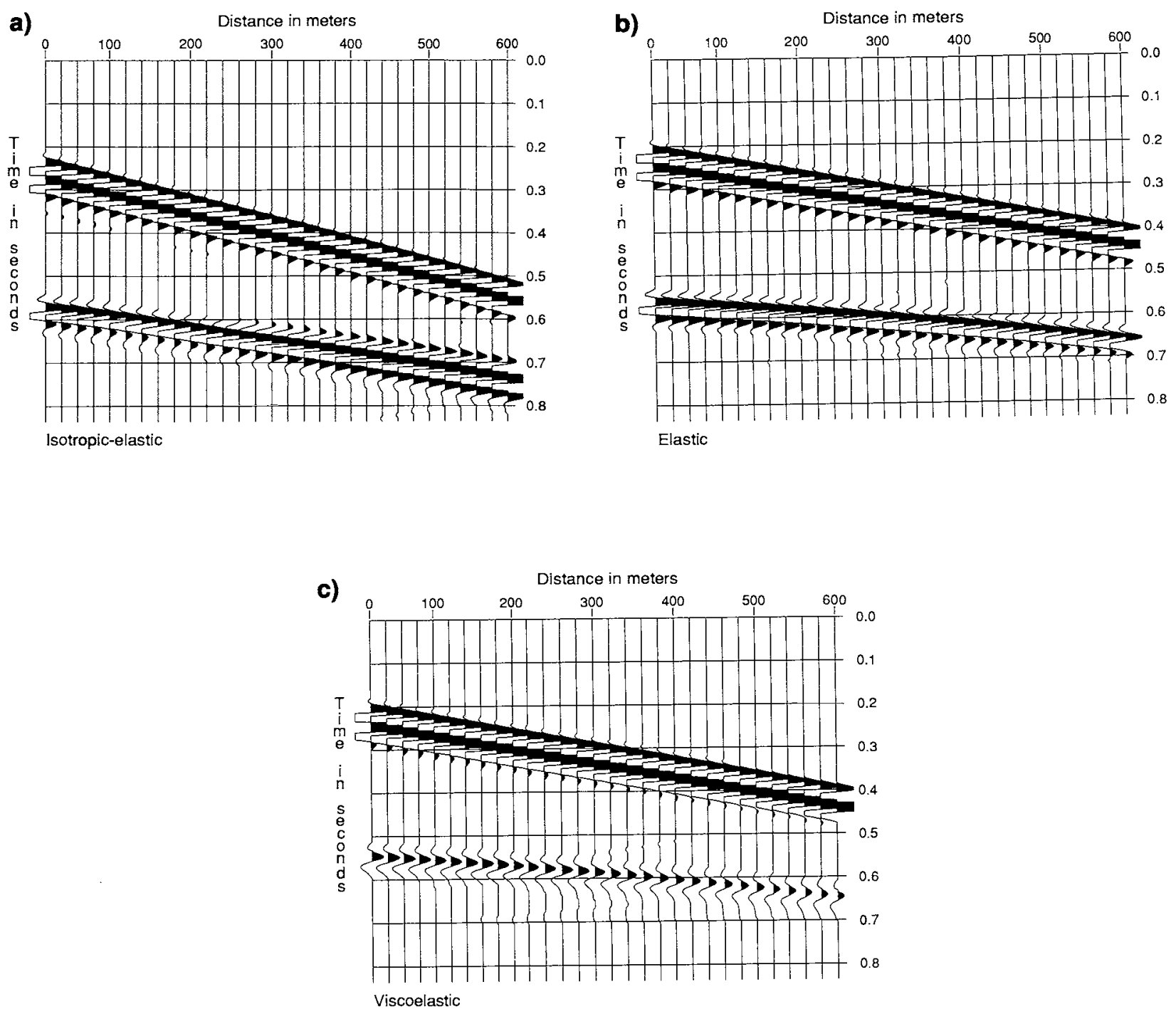

FIG. 3. Synthetic seismograms (not scaled) corresponding to (a) the isotropic-elastic case, (b) the anisotropic-elastic case, and (c) the anisotropic-viscoelastic case. The reflected anelastic wave shows an amplitude and phase behaviour different from that of the reflected elastic wave. Traveltime differences can also be appreciated.

FIg. 2. Snapshots of the displacement field (relative amplitudes) produced by a line source with a dominant frequency of $25 \mathrm{~Hz}$. In all the snapshots, the lower medium is isotropicelastic; analogously, in the figures that compose the left column, the upper medium is also isotropic-elastic, but with different material parameters. In the right column, the upper medium is anisotropic-viscoelastic. In the central column, the upper medium is anisotropic-elastic and corresponds to the low-frequency limit of the viscoelastic case. The triangle indicates the position of the first receiver. 